\title{
Fractional anisotropy helps predicts memory rehabilitation outcome after traumatic brain injury
}

\author{
Gary E. Strangman ${ }^{\mathrm{a}, \mathrm{b}, *}$, Therese M. O’Neil-Pirozzi ${ }^{\mathrm{b}, \mathrm{c}}$, Christina Supelana $^{\mathrm{a}, \mathrm{b}}$, Richard Goldstein ${ }^{\mathrm{b}}$, \\ Douglas I. Katz ${ }^{\mathrm{d}, \mathrm{e}}$ and Mel B. Glenn ${ }^{\mathrm{b}, \mathrm{f}}$ \\ ${ }^{a}$ Department of Psychiatry, Harvard Medical School, MA, USA \\ ${ }^{\mathrm{b}}$ Department of Physical Medicine and Rehabilitation, Spaulding Rehabilitation Hospital, MA, USA \\ ${ }^{\mathrm{c}}$ Department of Speech-Language Pathology and Audiology, Northeastern University, MA, USA \\ ${ }^{\mathrm{d}}$ Department of Neurology, Boston University School of Medicine, MA, USA \\ ${ }^{\mathrm{e}}$ Braintree Rehabilitation Hospital, Braintree, MA, USA \\ ${ }^{\mathrm{f}}$ Department of Physical Medicine and Rehabilitation, Harvard Medical School, MA, USA
}

\begin{abstract}
Traumatic brain injury (TBI) commonly results in residual memory difficulties. Such deficits are amenable to cognitive rehabilitation, but optimal selection of rehabilitation interventions remains a challenge. We hypothesized that diffusion tensor imaging (DTI) could be used to predict which individuals were likely to benefit from a specific memory rehabilitation intervention. Thirty-seven individuals with TBI, of all severities, first underwent DTI scanning, along with 18 matched controls. Participants with TBI then attended a 12-session memory intervention emphasizing internal memory strategies (I-MEMS). Primary outcome measures (HVLT, RBMT) were collected at the time of DTI scanning, and both immediately and one month post-therapy. In contrast to typical neuroimaging analysis, fractional anisotropy (FA) was used to predict long-term outcome scores, adjusting for typical predictors (injury severity, age, education, time since injury, pretest score). FA of the parahippocampal white matter was a significant negative predictor of HVLT, while the anterior corpus callosum, left anterior internal capsule, and right anterior corona radiata were negative predictors of RBMT outcome. The importance of these predictors rivaled those of pretest scores. Thus, FA measures may provide substantial predictive value for other cognitive interventions as well. The reason why higher FA was associated with less successful response to cognitive intervention remains unclear and will require further study.
\end{abstract}

Keywords: Cognitive rehabilitation, memory, diffusion tensor imaging, behavioral neurology, semantic clustering, brain trauma, structural neuroimaging

\section{Introduction}

Despite a wide variety of causes of traumatic brain injury (TBI) and the potential for a broad spatial distribution of injury [36], TBI has commonly been associated with prototypical anatomical injuries and func-

${ }^{*}$ Corresponding author: Dr. Gary Strangman, Neural Systems Group, Department of Psychiatry, 149 13th St - Suite 2651, Charlestown, MA 02129, USA. Tel.: +1 617724 0662; Fax: +1 617 726 4078; E-mail: strang@nmr.mgh.harvard.edu. tional deficits. Anatomical injuries typically include traumatic axonal injury (TAI), focal contusions in frontotemporal areas, and damage in regions particularly vulnerable to excitotoxicity [45]. Behaviorally speaking, the long-term functional consequences of TBI include consistent deficits in memory, attention, and in tasks relying heavily on executive functioning $[12,13$, 35].

Quantitative brain measures have been suggested as a useful approach to investigate the contribution of specific brain regions to functional deficits [8]. Diffusion tensor imaging (DTI), based on magnetic resonance 
imaging (MRI), has been widely regarded as a promising candidate in both mild and more severe TBI, in large part because of its high sensitivity to microstructural alterations in white matter $[1,8,30,77]$. A variety of distinct measures can be generated from DTI scans, including the apparent diffusion coefficient (ADC), fractional anisotropy (FA), oriented diffusivities, and various measures based on fiber tract tracing methods [3]. The most common DTI measure is fractional anisotropy (FA), which provides a single-number summary of the diffusion tensor of data at each voxel. FA values range between 0 and 1 and represent how "directional" water can diffuse within that voxel or region. Non-directional diffusion (as in a glass of water or a ventricle) would provide an $\mathrm{FA}=0$. In cases where all water is restricted to diffusing in a single direction, $\mathrm{FA}=1$. Myelinated axons provide substantial barriers to water diffusion and hence in major fiber bundles FA is high (e.g., in the corpus callosum, $\mathrm{FA} \approx 0.80)$, whereas in the ventricles FA is low (0.05 or less).

Interest in DTI imaging of individuals with TBI has grown dramatically in recent years. To date, the various diffusion measures have been used to assess three main types of questions. The first question relates to what sorts of white matter changes occur following TBI. Numerous studies have demonstrated widespread decreases in FA in individuals with TBI versus controls, particularly in midline, frontal, and temporal regions, most often in severe TBI [7,49,67,70,75,77]. However, some studies have reported increased FA. This begs the second type of question, namely how do white matter changes evolve over time. Wilde et al. [73] found increased FA in children with mild TBI less than six days post-injury. Two studies have found that FA in frontal and temporal tracts decreased between a few weeks post-injury and a year post-injury in individuals with severe TBI $[6,22]$. Sidaros and colleagues found both increases and decreases in FA between 8 weeks and 12 months following severe TBI, proposing possible regrowth of axons in this period to explain the FA increases [51]. In the corpus callosum, a significant reduction in FA was identified in the corpus callosum between one week and six months post-injury specifically in the four patients with the worst outcome [32].

The third line of questioning is whether white matter changes are related to current cognitive status or can predict general clinical outcomes. Numerous studies have demonstrated relationships between the extent of neuroanatomical injury and general TBI outcome $[2,4$, $25,28,44,72]$. Studies of white matter have found correlations between overall white matter disruption and general clinical outcome $[38,44,64,67,68]$, between FA and concurrent WAIS, Trails and Wechsler Memory Scale [62], between FA in the cerebral peduncle and the 1-year Glasgow outcome scale [51], between FA and concurrent cognitive processing speed, interference resolution and global outcome [31], as well as positive correlations between FA and both working and declarative memory measures [43]. Generally speaking, more severe injury is associated with worse outcome and poorer performance on neuropsychological and cognitive tests, although there are notable exceptions [23, 51].

Less well understood, however, is the relationship between neuroanatomical injury and outcome of a specific rehabilitation intervention. Information is particularly limited in people with chronic TBI, where substantially fewer clinical rehabilitation studies have been conducted, perhaps due to typical reimbursement processes. TBI rehabilitation interventions have been shown to help improve memory and executive function [13], attention [27], community integration [14] and functional outcomes [21]. Individual variation in outcome is notable. However, very little research has been performed to predict outcomes from specific rehabilitation interventions prior to their initiation, and no neuroimaging results have been reported in this domain.

In our previous studies of chronic TBI, we showed that both task-related brain activation and regional brain volumes provide value for predicting memory rehabilitation outcome $[59,60]$. However, no prior studies have examined the ability of DTI-related measures to predict outcome from a TBI rehabilitation intervention. We therefore sought to test whether a key measure of white matter integrity (FA) derived from DTI scans could predict outcomes from a TBI memory rehabilitation intervention. Our rehabilitation intervention was the I-MEMS protocol, designed to improve participants' memory by training individuals to use internally-based semantic association and related strategies [41].

Previously published DTI analysis approaches have generally fallen into one of three categories. The first involves region-of-interest (ROI) analysis in which an operator manually outlines (or, in some cases, uses an atlas to define) specific regions in each individual $[28,75]$. Summary statistics are then computed for each region in each individual, which can be used in group statistical analysis. Second, fiber tractography has been utilized to measure the integrity of white matter tracts [62,71]. This also requires a process for defining ROIs, but provides a slightly less operator- 
dependent way to assess the connectivity between or passing through given ROIs, as well as computing FA or ADC. A third approach is voxel-based analysis of whole-brain data, typically examining FA or ADC values $[43,49]$.

Voxel-wise analyses do not require an operator and hence are minimally affected by analyst bias. However, a well-recognized challenge in voxel-wise FA analysis is the difficulty in co-registering FA maps across individuals [53]. White matter tracts are highly variable from subject to subject, and small errors in alignment can result in misleading findings. Such errors are likely exaggerated in patient populations with structural brain alterations (e.g., severe TBI, stroke). One way to address this limitation is tract-based assessment, as with FSL's Tract Based Spatial Statistics, TBSS [53]. In TBSS, FA maps for all individuals are first aligned reasonably closely via a non-linear warping algorithm. Then, to compensate for any residual errors in registration, the center of tracts are identified and the maximal FA value perpendicular to that tract is projected onto the tract. In this way, the peak FA from an offcenter (or mis-registered) tract from one individual is "pulled" back to the main tract to improve comparison with other subjects [53]. TBSS can thus provide a more robust method for alignment of FA maps in groups that include structural brain injury.

Once such alignment is complete, previous studies have sought to explain the DTI data in terms of demographic or functional variables (e.g., age, Glasgow Coma Scale score, injury severity, and so on). In contrast, when selecting rehabilitation interventions the question of interest is whether DTI data can predict the outcome from a yet-to-be-implemented intervention. This requires using the DTI data as a predictor in modeling, rather than as an outcome variable. For whole-brain (voxel wise) analysis, existing neuroimaging packages are not designed to perform this type of computation. Therefore, we implemented a novel approach using whole-brain FA maps to investigate the regional specificity in the ability of FA to predict rehabilitation outcomes from our I-MEMS therapy.

\section{Materials and methods}

\subsection{Participants}

Participants with TBI were recruited via mailings to clients of the Massachusetts Rehabilitation Commission's Brain Injury and Statewide Specialized Com- munity Services, members of the Brain Injury Association of Massachusetts, local support groups, and to patients of study-affiliated physicians. Some 58 individuals were initially enrolled, following a procedure approved by the Human Research Committee at the Massachusetts General Hospital. All participants (1) were at least 18 years of age at the time of injury, (2) had sustained a TBI of any severity at least 12 months prior to the study, (3) were right-handed [42] and fluent in English, and (4) self-reported as having difficulty with memory following their injury. Exclusion criteria included a score of less than 4 on either the expression or comprehension items of the FIM, non-traumatic etiology of cerebral dysfunction in addition to TBI, active major illnesses, pre-injury history of psychiatric disease, inability to read single words at an eighth-grade reading level, and current drug or alcohol dependence (criteria from the Diagnostic and Statistical Manual of Mental Disorders, 4th Edition).

From this group, the first 16 subjects underwent only a limited (6-direction) DTI data collection protocol and hence were excluded from further analysis. Data was also excluded from four individuals who failed to complete at least 8 of 12 memory intervention sessions and/or return for post-testing. Finally, technical difficulties for one participant's scans led to insufficient image quality to attempt analysis. We proceeded to analyze the remaining 37 participants with TBI (11 female, 26 male).

In addition, a group of 21 healthy, right-handed control participants was matched to the participants with TBI. In two controls, we were unable to complete the scanning procedure. Technical issues with the scanner precluded saving data from a third, so we proceeded with analysis of $n=18$ controls. Demographic information for both study groups appears in Table 1, and injury and severity information for participants with TBI appear in Table 2.

These same participants were part of a larger TBI Model Systems research project, for which publications on related topics have appeared previously [41,57-60].

\subsection{Experimental procedures}

The study consisted of 16 separate sessions, as described previously [59]. To summarize, Session 1 involved a battery of standard neuropsychological tests of memory, executive function, and language. Tests included Hopkins Verbal Learning Test-Revised (HVLTR), the Rivermead Behavioral Memory Test (RBMTII), the Boston Naming Test (BNT) 2nd Edition Short 
Table 1

Demographic characterization of study participants

\begin{tabular}{|c|c|c|c|c|c|c|c|c|c|}
\hline & \multicolumn{4}{|c|}{ Participants with TBI $(n=37)$} & \multicolumn{4}{|c|}{ Control participants $(n=18)$} & \multirow[b]{2}{*}{$\mathrm{p}$} \\
\hline & Mean & $\mathrm{SD}$ & Min & Max & Mean & SD & Min & Max & \\
\hline Age & 45.7 & 11.2 & 24.3 & 64.9 & 45.9 & 9.0 & 23.9 & 58.5 & 0.93 \\
\hline Education (years) & 14.6 & 2.2 & 10 & 20 & 15.4 & 2.5 & 12.0 & 20.0 & 0.16 \\
\hline
\end{tabular}

Table 2

Injury characteristics of participants with TBI

\begin{tabular}{lccc}
\hline & \multicolumn{3}{c}{ TBI Participants $(n=37)$} \\
\cline { 2 - 4 } Characteristics & Mean (SD) & Min & Max \\
\hline Time since injury (y) & $13.9(17.4)$ & 1.3 & 37.6 \\
Loss of consciousness (d) & $14.7(26.5)$ & 0 & 105 \\
& Mild & Moderate & Severe \\
Injury Severity & $10(27 \%)$ & $7(19 \%)$ & $20(54 \%)$ \\
\hline
\end{tabular}

Form, the Boston Diagnostic Aphasia Examination (BDAE) 3rd Edition Animal Naming, Trail Making Test Part A and B, and Wechsler Memory ScaleRevised Digit Span (Forward and Backward). In Session 2, approximately three days later, each study participant underwent MRI neuroimaging, including structural, diffusion, and functional scanning, described in more detail below.

Sessions 3 through 14 were memory intervention sessions led by one of the investigators. This intervention emphasized internal memory strategy training and were designed around evidence-based approaches [41]. We utilized a group intervention with 3 to 6 members per group cycle and 2 to 3 group facilitators. Each group cycle ran twice weekly for 6 weeks, 90 minutes per session, for a total of 12 sessions. Sessions emphasized semantic association and other internally based strategies (e.g., elaboration and imagery) from encoding, storage, and retrieval perspectives. Session 15 (posttest 1) was conducted within 3 days after the final memory intervention session and repeated all of the tests from session 1 except for the BNT and BDAE.

Session 16 (posttest 2) was conducted 1 month following session 15 and again repeated the same tests. No MRI scanning was performed at either posttest session. Forms of the various tests were counterbalanced across participants and sessions. Pre- and posttest evaluations were conducted by different individuals, with regular cross-validation of test administrators.

Outcome variables, measured at pretest and both posttest time points, included the HVLT-R [10] and the RBMT-II [76]. The HVLT was a primary outcome because it is widely used, it emphasizes semantic association memory (a focus of the I-MEMS intervention), and it has multiple parallel versions to help minimize practice effects. The RBMT was used as an ecologically valid, broad measure of impairment in everyday mem- ory functioning, also with multiple forms for repeated testing.

\subsection{MRI data acquisition}

All MRI was performed with a Siemens Avanto 1.5 Tesla scanner with 8-channel Tim head-coil. Two MPRAGE sequences were collected for high-resolution anatomy $(\mathrm{TR}=1.91 \mathrm{~s}$, TE $=4.13 \mathrm{~ms}$, TI $=1.1 \mathrm{sec}$, flip angle $=15 \mathrm{deg}, 120$ slices, matrix $128 \times 128,1 \times$ $1 \times 1 \mathrm{~mm}$ resolution). We then performed a single DTI scan (30 directions, low-/high-b $=0,1000 \mathrm{~s} / \mathrm{mm}^{2}$, TR $=5.0 \mathrm{~s}, \mathrm{TE}=84 \mathrm{~ms}, 23$ slices, $1.7 \times 1.7 \times 5.0 \mathrm{~mm}$ voxels). Functional MRI and clinical scans (T2-weighted, FLAIR and hemosiderin) were also collected, the results of which are treated in separate publications.

\subsection{Data analysis}

Neuropsychological tests were scored per respective standards. The two rehabilitation outcome measures we considered were (1) the HVLT delayed correct recall score (range: 0-12), and (2) the RBMT total standardized profile score (range: 0-24). Injury severity was categorized as mild, moderate or severe based on a method used in our previous studies [59]. Briefly, 85\% of cases were based on duration of loss of consciousness (LOC) [16,26,47], where mild was 0-30 min LOC (unless post traumatic amnesia exceeded 24 hours); moderate was $30 \mathrm{~min}<\mathrm{LOC} \leqslant 24$ hours, and $>24$ hours LOC was designated severe. In the absence of LOC data, the determination was made based on Glasgow coma scale scores, where $<9=$ severe, $9-12=$ moderate, and $13-15=$ mild $[26,63]$.

Voxelwise statistical analysis of the DTI data was carried out using the TBSS package [53] from FSL v4.1.2 [54]. First, FA images were created by fitting a tensor model to the raw diffusion data using FSL's FDT. These images then were resliced to $2 \times 2 \times$ $2 \mathrm{~mm}$ resolution using sinc interpolation, eddy corrected, and brain-extracted [52]. All subjects' FA data were smoothed with a 5mm FWHM kernel (consistent with the largest acquisition dimension) and aligned into a common space using FSL's nonlinear registration tool FNIRT, which uses a b-spline representation of the reg- 


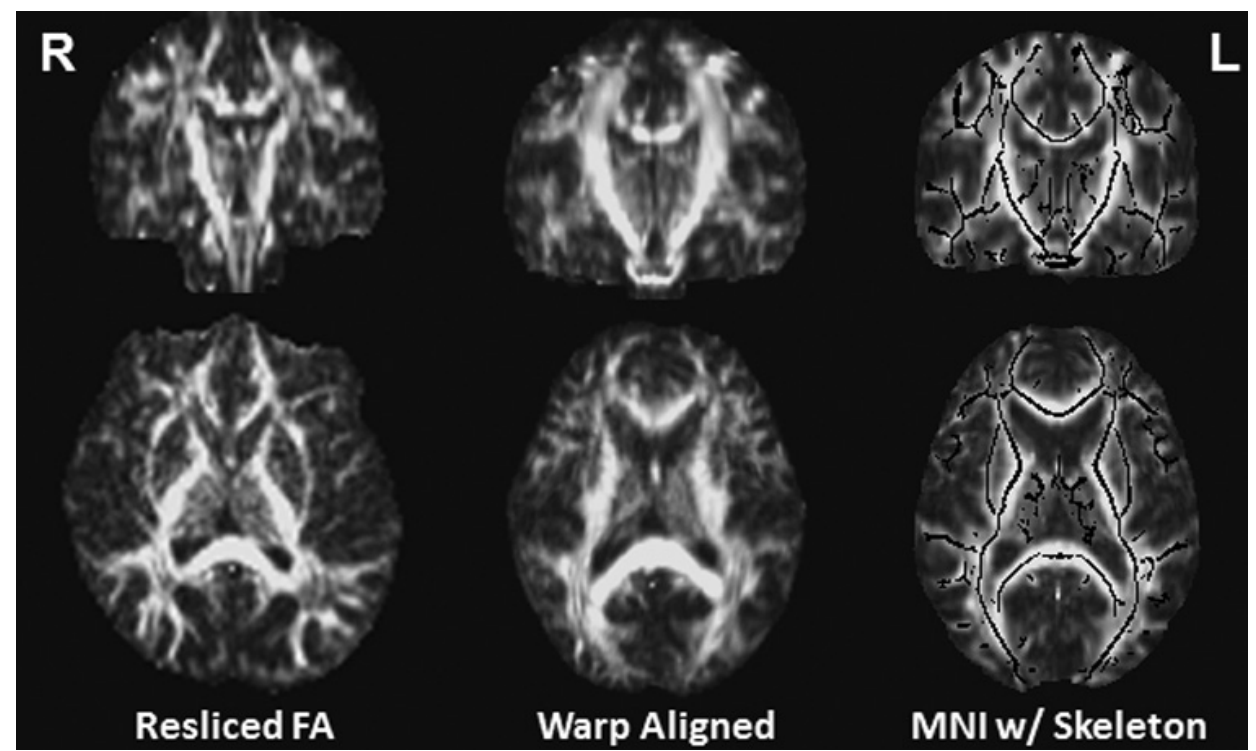

Fig. 1. Example DTI FA analysis process for a participant with TBI using the FSL-TBSS pipeline. FA maps were generated via FSL and resliced to $2 \mathrm{~mm}$ isotropic voxels (left), and then were non-linearly aligned to one another (center). To improve inter-subject alignment, a mean FA skeleton was generated from all participants (black lines, right) onto which the maximum FA values from the surrounding tracts were projected, to improve inter-subject alignment.

istration warp field [46]. Next, results were manually examined for qualitative failures to normalize or register the FA images. No such failures were observed. The mean FA image across all 55 subjects was computed and thinned to create a mean FA skeleton, representing the centers of all tracts common to the group. Each subject's warped FA data was then projected onto this skeleton for final alignment [53]. The resulting skeleton maps (one per subject) were used for voxelwise between-subject analyses as described below.

\subsection{Cross sectional and severity analysis}

Participants with TBI $(n=37)$ were compared to matched controls $(n=18)$ via a simple T-test procedure comparing the individual FA skeletons. Correction for multiple comparisons across voxels in the skeleton was achieved using the Threshold-Free Cluster Enhancement (TFCE) method available via FSL's randomise tool. This technique enables correction for multiple comparisons to $p<0.05$ based on the number of voxels in a cluster without having to pre-specify a cluster-forming threshold [55]. We report only cross sectional findings that pass this correction for multiple comparisons. All 55 participants were also entered into a severity regression analysis, whereby FA was regressed against three indicator variables: mild, moderate, and severe TBI (controls serving as a reference group) to identify regions of significant change in FA as a function of injury severity.

\subsection{Prediction modeling}

For the 37 individuals with TBI, we then used linear mixed effects regression models to predict posttest outcome based on the following predictors: pretest outcome, age, time since injury, education, moderateTBI, severeTBI, and regional FA. Posttest outcome was either the HVLT delayed correct recall score or RBMT total standardized profile score at posttest 2 (i.e., one month after completion of therapy). Pretest outcome was the same outcome at pretest, age was the participant's age in years at the time of the MRI, education and time since injury were in years, and regional FA for each voxel was the FA value found at that point in the mean FA skeleton. ModerateTBI and severeTBI were again binary indicator variables ,marking whether a participant had sustained a moderate or severe injury, as determined from Glasgow Coma Scale and length of loss of consciousness data [59]. This model was fit voxel-by-voxel for both HVLT and RBMT. We focused on posttest 2 as our behavioral work has demonstrated the I-MEMS therapy provides significant and lasting improvements in memory function [41], and we were most interested in predictors of lasting outcome. For each outcome, we also fit two additional models: one 


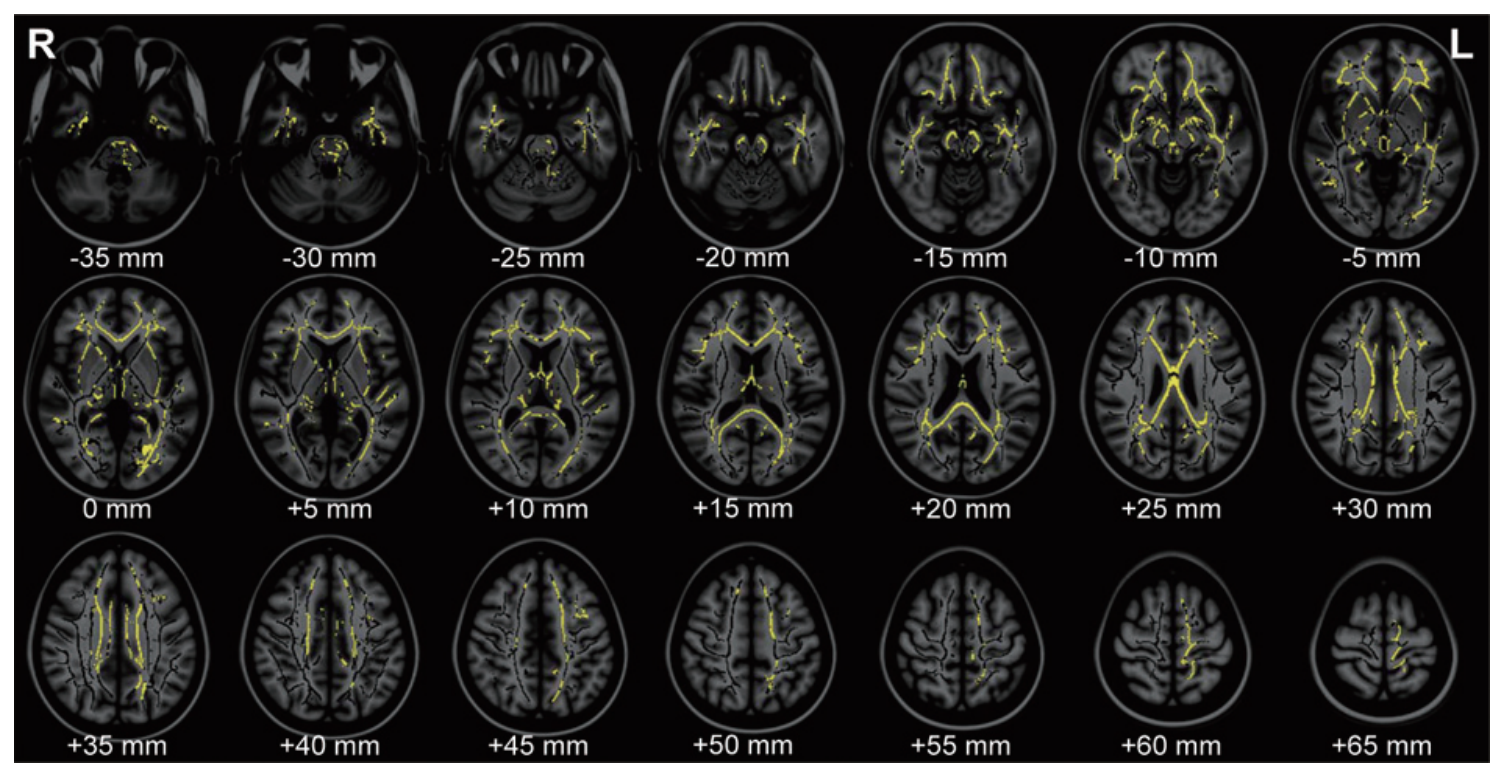

Fig. 2. Regions where participants with TBI<control FA (yellow), overlaid on the MNI Colin template. Black lines indicate portions of the mean FA skeleton where no significant FA differences were observed between groups. (Colours are visible in the online version of the article; http://dx.doi.org/10.3233/NRE-2012-0797)

using only FA plus pretest score on the outcome variables as predictors, and one using FA alone to predict outcome. These were used to determine the potential influence of the other model predictors in the full model (e.g., collinearity relations such as masking, suppression and error inflation).

Standard neuroimaging data analysis packages (FSL, SPM, AFNI, others) only allow MRI measures to be a dependent variable, not an independent variable as we sought here. We thus performed our analysis using custom Python code (with the PypeR module) to deliver all demographic and FA data for a given voxel to the R statistics package (v2.11), compute the model in question, return and save the results in Python, and continue to the next voxel [61]. The resulting statistical maps on the FA skeleton were then corrected for multiple comparisons using AFNI's 3dClustSim program identify extent thresholds that met a false positive error rate of $p<0.05$, using a voxelwise clustering-forming threshold of $p<0.002$.

\section{Results}

\subsection{Participant characterization and behavioral results}

Injury characteristics for study participants appear in Tables 2 and 3. Many of the brain injuries were remote
Table 3

Patients taking anticonvulsant medications, and cause of injury information for participants with TBI

\begin{tabular}{lc}
\hline Condition & Participants \\
\hline Anticonvulsant medications & $4(11 \%)$ \\
Cause of Injury & \\
Vehicle accident & $24(65 \%)$ \\
Blunt force trauma & $6(16 \%)$ \\
Fall & $5(14 \%)$ \\
Mixed & $2(5 \%)$ \\
\hline
\end{tabular}

( $>10$ years), and over half were severe. The outcome measures at all three time points for TBI participants appear in Table 4, along with the results of simple paired T-tests of posttest versus pretest scores. Per Table 4, the 37 participants with TBI demonstrated significant improvements for both semantic memory (HVLT) and everyday memory function (RBMT) at posttest 1 and 2 (i.e., immediate and delayed time points), similar to our previous report [41]. Thus, outcomes were significantly improved by rehabilitation immediately after therapy, and these improvements were retained over the 1-month delay re-test interval.

\subsection{TBI versus control FA}

Results when comparing the skeletonized FA in our participants with TBI versus matched controls appear in Fig. 2. First, the extent of disruption associated with TBI in our population was substantial, affecting most 
Table 4

Outcome scores for TBI participants $(n=37)$ measured at three time points, with statistical comparisons against pretest scores

\begin{tabular}{|c|c|c|c|c|}
\hline \multirow[b]{2}{*}{ Time Point } & \multicolumn{2}{|c|}{ HVLT-R delayed recall } & \multicolumn{2}{|c|}{ RBMT-II total score } \\
\hline & Mean (SD) & $\mathrm{T}(\mathrm{p})$ & Mean (SD) & $\mathrm{T}(\mathrm{p})$ \\
\hline Pretest & $6.4(3.9)$ & - & $16.3(4.7)$ & - \\
\hline Posttest 1 (Immediate) & $8.4(3.2)$ & $4.3(0.0001)$ & $18.2(4.5)$ & $3.8(0.0006)$ \\
\hline Posttest 2 (1-month follow-up) & $8.6(3.1)$ & $5.8(<0.0001)$ & $17.9(4.2)$ & $3.2(0.003)$ \\
\hline \multicolumn{5}{|c|}{$\begin{array}{l}\text { Table } 5 \\
\text { Regions of white matter where FA for controls }>\text { TBI }\end{array}$} \\
\hline Region & & & Left & Right \\
\hline \multicolumn{2}{|c|}{ Corpus callosum (genu, body, splenium) } & & - & - \\
\hline \multicolumn{2}{|c|}{ Cingulum } & & - & - \\
\hline \multicolumn{2}{|l|}{ Fornix } & & - & - \\
\hline \multicolumn{2}{|l|}{ Cerebral peduncles } & & - & - \\
\hline \multicolumn{2}{|l|}{ Corona radiata (anterior) } & & - & - \\
\hline \multicolumn{2}{|l|}{ Corona radiata (superior) } & & - & - \\
\hline \multicolumn{2}{|l|}{ Corona radiata (posterior) } & & - & - \\
\hline \multicolumn{2}{|l|}{ Uncinate fasciculus } & & - & - \\
\hline \multicolumn{2}{|c|}{ External capsule } & & - & - \\
\hline \multicolumn{3}{|c|}{ Sagittal stratum (inferior longitudinal and fronto-occipital fasciculus) } & - & $\mathrm{O}$ \\
\hline \multicolumn{2}{|c|}{ Internal capsule, anterior } & & - & $\mathrm{O}$ \\
\hline \multicolumn{2}{|l|}{ Thalamic radiation } & & $\mathrm{O}$ & - \\
\hline
\end{tabular}

major tracts, and with regions being affected largely bilaterally as listed in Table 5. Second, we did not identify any regions where participants with TBI had significantly higher FA than controls $(p>0.5$ throughout the brain). Third, while many tracts with altered FA were quite symmetric, there was a bias towards left-sided fiber tract disruption in select areas: (i) multiple tracts superior to $\mathrm{MNIz}=+40 \mathrm{~mm}$ which includes the superior corpus callosum, corticospinal and corticopontine tracts, the superior corona radiata and the superior thalamic radiation, (ii) the sagittal stratum underlying the left superior temporal gyrus, (iii) the left posterior thalamic/optic radiation (underlying the left middle occipital and lingual gyri), (iv) the left superior cerebellar peduncle, and the (v) pontine crossing tract. Finally, the regions of significant FA reductions following TBI did not comprehensively cover the mean FA skeleton (116,832 voxels in all), but were instead restricted to approximately a third of the skeleton (38,577 voxels). As is evident in Fig. 2, more superior and more lateral tracts were generally spared, including the superior longitudinal fasciculus, as well as much of the internal capsule, and the sagittal stratum (including the inferior longitudinal fasciculus and inferior fronto-occipital fasciculus, particularly on the right).

\subsection{FA versus injury severity}

In our analysis of injury severity, we identified no significant differences in FA for individuals with mild injuries $(n=10)$ relative to controls, four clusters of voxels with reduced FA for individuals with moderate injuries $(n=7)$, and 32 clusters of voxels for individuals with severe injuries $(n=20)$. Details on locations and cluster sizes appear in Table 6.

When considering all voxels in the FA skeleton (not just those significantly different from controls), $28 \%$, $33 \%$ and $20 \%$ of the differences were FA increases relative to controls for mild, moderate and severe subjects, respectively. Based on bionomial tests, this variation was significantly different both from a prediction of random changes (50\% probability of FA increases), as well as from an assumption of exclusively FA reductions ( $0 \%$ probability of FA increases), $p<0.00001$ for each severity.

\subsection{Prediction of rehabilitation outcome}

For prediction of rehabilitation outcome, we fit two primary models: one to predict HVLT-R outcome at posttest 2 and one to predict the RBMT-II outcome at posttest 2. These were fit at every voxel in the mean FA skeleton to identify regions of white matter that are predictive of outcome from our I-MEMS memory therapy. In each model, we found that (1) the pretest predictor (HVLT-R or RBMT-II) was significant at every voxel, as expected, (2) FA was a significant predictor in some voxels and not others, as elaborated below, and (3) none of the other model terms reached significance 
Table 6

Regions of FA where participants with TBI $<$ controls by injury severity

\begin{tabular}{|c|c|c|c|c|c|c|}
\hline Location & \# voxels & Hemi & MNIx & MNIy & MNIz & Tmax \\
\hline \multicolumn{7}{|l|}{ Mild } \\
\hline \multicolumn{7}{|l|}{ No clusters found } \\
\hline \multicolumn{7}{|l|}{ Moderate } \\
\hline Fornix & 91 & mid & -2 & -3 & 10 & -4.33 \\
\hline Corpus callosum, mid & 82 & $\mathrm{R}$ & 6 & 0 & 26 & -3.84 \\
\hline Cingulum, mid & 81 & $\mathrm{R}$ & 18 & -8 & 39 & -4.87 \\
\hline Corona radiata, posterior & 61 & $\mathrm{~L}$ & -29 & -50 & 20 & -5.25 \\
\hline \multicolumn{7}{|l|}{ Severe } \\
\hline Corpus callosum, posterior & 1882 & $\mathrm{~L}$ & -8 & -26 & 26 & -5.68 \\
\hline Fornix & 829 & mid & -2 & -5 & 10 & -7.31 \\
\hline Corona radiata, anterior & 731 & $\mathrm{~L}$ & -20 & 38 & 10 & -4.98 \\
\hline Cingulum, mid & 640 & $\mathrm{R}$ & 18 & -8 & 39 & -5.88 \\
\hline External capsule & 597 & $\mathrm{~L}$ & -35 & -10 & -8 & -4.99 \\
\hline Inferior fronto-occipital fas. & 502 & $\mathrm{R}$ & 15 & 37 & -14 & -4.81 \\
\hline Corona radiata, posterior & 292 & $\mathrm{R}$ & 29 & -54 & 24 & -4.74 \\
\hline Thalamus & 196 & $\mathrm{R}$ & 18 & -33 & 6 & -6.57 \\
\hline Cerebral peduncle & 181 & mid & 0 & -23 & -11 & -6.01 \\
\hline Sagittal stratum & 176 & $\mathrm{R}$ & 41 & -15 & -13 & -5.65 \\
\hline Inferior fronto-occipital fas. & 127 & $\mathrm{~L}$ & -18 & 19 & -15 & -4.67 \\
\hline Uncinate fasciculus & 122 & $\mathrm{R}$ & 26 & 19 & -18 & -4.71 \\
\hline Thalamic radiation, posterior & 122 & $\mathrm{~L}$ & -33 & -51 & 18 & -4.88 \\
\hline Uncinate fasciculus & 112 & $\mathrm{~L}$ & -25 & 24 & -12 & -5.59 \\
\hline Thalamus & 111 & $\mathrm{~L}$ & -4 & -17 & -1 & -5.32 \\
\hline Cingulum, mid & 111 & $\mathrm{~L}$ & -6 & -7 & 37 & -5.15 \\
\hline Cingulum, inferior & 100 & $\mathrm{~L}$ & -31 & -3 & -28 & -5.03 \\
\hline Inferior fronto-occipital fas. & 100 & $\mathrm{~L}$ & -9 & 46 & -19 & -4.67 \\
\hline Superior longitudinal fas. & 92 & $\mathrm{~L}$ & -41 & 9 & 15 & -6.61 \\
\hline Thalamic radiation, posterior & 91 & $\mathrm{~L}$ & -24 & -73 & 1 & -4.37 \\
\hline Corona radiata, anterior & 90 & $\mathrm{~L}$ & -30 & 32 & -4 & -4.50 \\
\hline Corpus callosum, mid & 84 & $\mathrm{~L}$ & -18 & 3 & 47 & -4.10 \\
\hline Corona radiata, anterior & 81 & $\mathrm{R}$ & 40 & 18 & 19 & -4.45 \\
\hline Corpus callosum, genu & 69 & $\mathrm{R}$ & 10 & 27 & 14 & -3.86 \\
\hline Pontine crossing tract & 68 & $\mathrm{~L}$ & -4 & -30 & -31 & -5.10 \\
\hline Cingulum, inferior & 68 & $\mathrm{~L}$ & -37 & -16 & -29 & -5.97 \\
\hline Middle cerebellar peduncle & 67 & $\mathrm{~L}$ & -6 & -19 & -33 & -3.97 \\
\hline Thalamus & 67 & $\mathrm{R}$ & 21 & -23 & 2 & -4.81 \\
\hline Corona radiata, posterior & 65 & $\mathrm{R}$ & 23 & -40 & 29 & -4.16 \\
\hline External capsule & 63 & $\mathrm{R}$ & 25 & 18 & -8 & -4.05 \\
\hline Stria terminalis & 57 & $\mathrm{R}$ & 25 & -31 & 0 & -5.30 \\
\hline Cerebral peduncle & 55 & $\mathrm{R}$ & 9 & -17 & -18 & -4.07 \\
\hline
\end{tabular}

for predicting either HVLT or RBMT outcome from our I-MEMS therapy in more than isolated voxels. Recall that these other model terms included age, education, injury severity, and time since injury.

For HVLT-R, and after correction for multiple comparisons, two symmetric regions exhibited significant rehabilitation outcome prediction based on FA: the left and right parahippocampal gyri (see Fig. 3A), superior to the hippocampus proper with peaks at MNI coordinates $[-23,2,-12]$ and $[26,-7-11]$, and peak $\mathrm{T}$ values of -5.1 and -4.9 respectively. Coefficients for the FA term in both regions were negative, implying that higher FA values were associated with lower HVLT scores at posttest 2, one month post-therapy (Fig. 3B).

In Table 7 we show the results of the regression con- ducted at the peak value of the FA coefficient, which resided within the right parahippocampal region. The only significant predictors of outcome were pretest HVLT and the regional FA. Given the range of FA values observed in this voxel $(0.512-0.158=0.355)$, the regression coefficient for FA explained a range of $0.355^{*} 25.2=8.9$ points on the HVLT outcome. This was slightly larger than the range predicted by the pretest HVLT scores, which predicted a range of (12$0)^{*} 0.6=7.2$ points in HVLT at posttest. While these quantitative details changed slightly at different voxels within the two identified clusters, the results remained qualitatively the same: FA and pretest HVLT scores were the only predictors of posttest HVLT at 1 month post-therapy. 
Table 7

Regression results from the right parahippocampus predicting HVLT at posttest 2

\begin{tabular}{|c|c|c|c|c|c|c|}
\hline Region [FA range] & Variable & Coef. (SE) & CI-low & CI-high & $\mathrm{T}$ & $\mathrm{p}$ \\
\hline R Parahippocampus & $\mathrm{FA}$ & $-25.2(5.3)$ & -36.0 & -14.5 & -5.1 & $<0.0001$ \\
\hline \multirow[t]{6}{*}[0.158-0.513]{} & preHVLT & $0.6(0.09)$ & 0.5 & 0.8 & 7.3 & $<0.0001$ \\
\hline & Age (y) & $0.007(0.03)$ & -0.05 & 0.06 & 0.3 & 0.79 \\
\hline & Education (y) & $-0.2(0.2)$ & -0.5 & 0.1 & -1.4 & 0.17 \\
\hline & ModerateTBI & $-1.5(0.02)$ & -3.3 & 0.3 & -1.7 & 0.11 \\
\hline & SevereTBI & $-0.9(0.7)$ & -2.3 & 0.6 & -1.2 & 0.23 \\
\hline & Sinceinjury (y) & $0.008(0.02)$ & -0.03 & 0.04 & 0.4 & 0.66 \\
\hline
\end{tabular}

${ }^{*}$ Coef. $=$ regression coefficient; $\mathrm{SE}=$ standard error of the coefficient; CI-low $=$ minimum value of the $95 \%$ confidence interval; CI-high $=$ maximum value of the $95 \%$ confidence interval; $\mathrm{T}, p=\mathrm{T}$-score and associated $\mathrm{p}$-value for this regression variable.
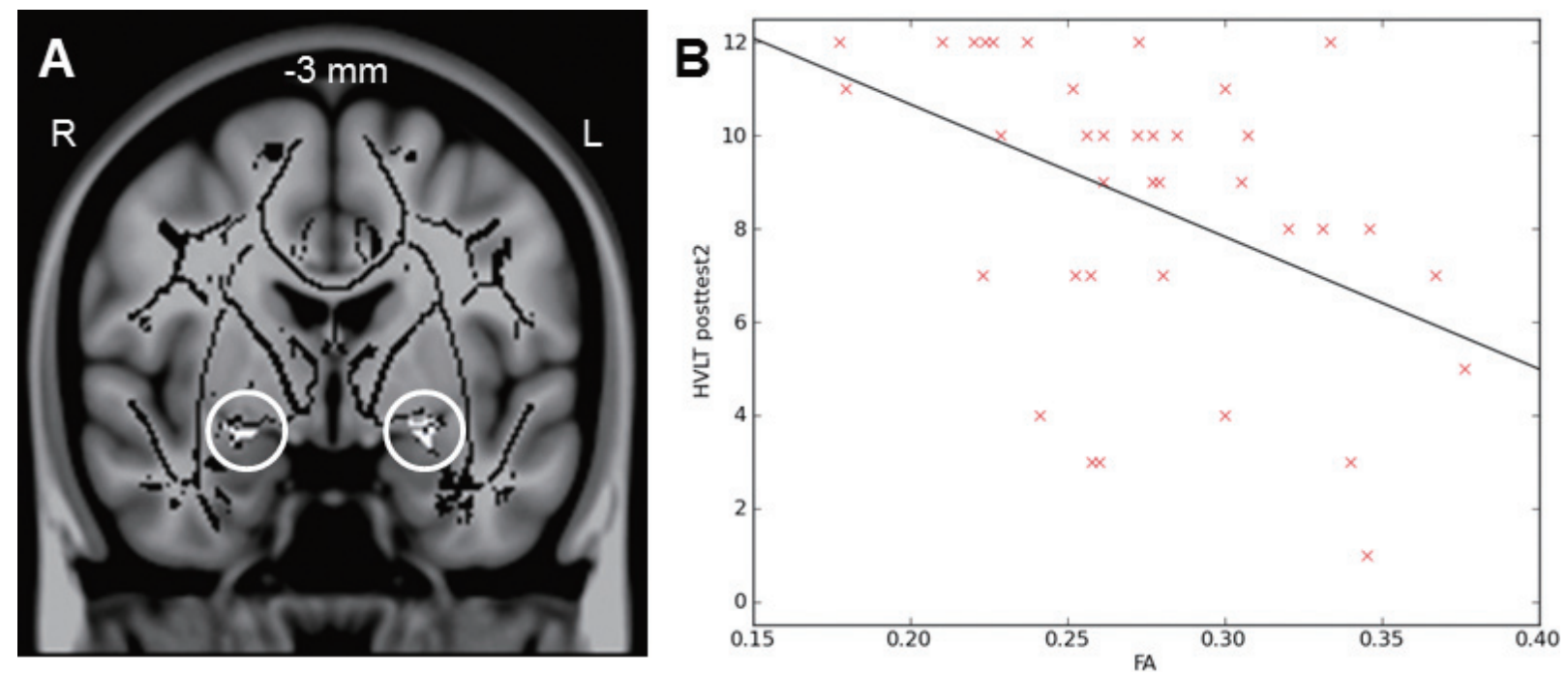

Fig. 3. (A) Bilateral parahippocampal regions (white) wherein FA was a significant predictor of HVLT outcome one month post-therapy (cluster-based correction for multiple comparisons at $p<0.05$ ). Black lines again represent the TBSS-generated FA skeleton. (B) Plot of HVLT outcome score versus FA for the peak voxel in the right parahippocampal cluster. A simple regression line is plotted; see Table 7 for results from the multiple regression model. (Colours are visible in the online version of the article; http://dx.doi.org/10.3233/NRE-2012-0797)

For the RBMT-II outcome, again after correction for multiple comparisons, three frontally located regions were significantly predictive of RBMT outcome at posttest 2: (1) the right anterior region of the corona radiata (Fig. 4A), the left anterior limb of the internal capsule (Fig. 4B), and the rostral body of the corpus callosum (Fig. 4C). As with the HVLT outcomes, all regression coefficients were negative, indicating that higher FA values in these regions were also associated with lower RBMT outcome scores.

In Table 8 we present the regression results for peak voxels in each of the three regions identified in Fig. 4. Data for only FA and pretest RBMT are presented as the other terms were not significant predictors of outcome. Also similar to HVLT, the RBMT pretest scores predicted 6.8-8.5 points of change on the RBMT outcome in these three regions (given a pretest RBMT score range of 6-23) whereas the FA term predicted a slightly higher 7.2-14.1 points of change on the RBMT.

We further note that when comparing the FA reduction maps in Fig. 2 to the regions found predictive of rehabilitation outcome (Figs 3 and 4), there was essentially no overlap. That is, there was no evidence of significant FA reductions relative to controls in any of the regions that provided predictive value for the two I-MEMS therapy outcome measures.

Finally, in our model checks, we computed prediction models using only FA as a predictor, and separate models using only FA and pretest score as predictors. In each case the coefficients on the FA predictors remained negative, strongly suggesting the negative signs on the regression parameters were not due to co-linearity problems such as suppression or masking. In addition, we did not identify any highly leveraged outliers based on Cook's distance assessment, indicat- 
Table 8

Outcome prediction regression results for RBMT at posttest 2

\begin{tabular}{clccccc}
\hline Region [FA range] & & Coef. (SE) & CI-low & CI-high & T & $\mathrm{p}$ \\
\hline Anterior corona radiata & FA & $-29.9(5.2)$ & -40.5 & -19.3 & -5.8 & $<0.0001$ \\
{$[0.228-0.698]$} & preRMBT & $0.5(0.1)$ & 0.3 & 0.8 & 4.7 & $<0.0001$ \\
Anterior limb of internal capsule & FA & $-27.2(5.8)$ & -39.2 & -15.3 & -4.7 & $<0.0001$ \\
{$[0.324-0.702]$} & preRBMT & $0.4(0.1)$ & 0.1 & 0.6 & 3.1 & 0.004 \\
Rostral body of corpus callosum & FA & $-11.8(3.5)$ & -19.1 & -4.6 & -3.3 & 0.002 \\
{$[0.164-0.777]$} & preRBMT & $0.5(0.1)$ & 0.2 & 0.7 & 3.3 & 0.003 \\
\hline
\end{tabular}
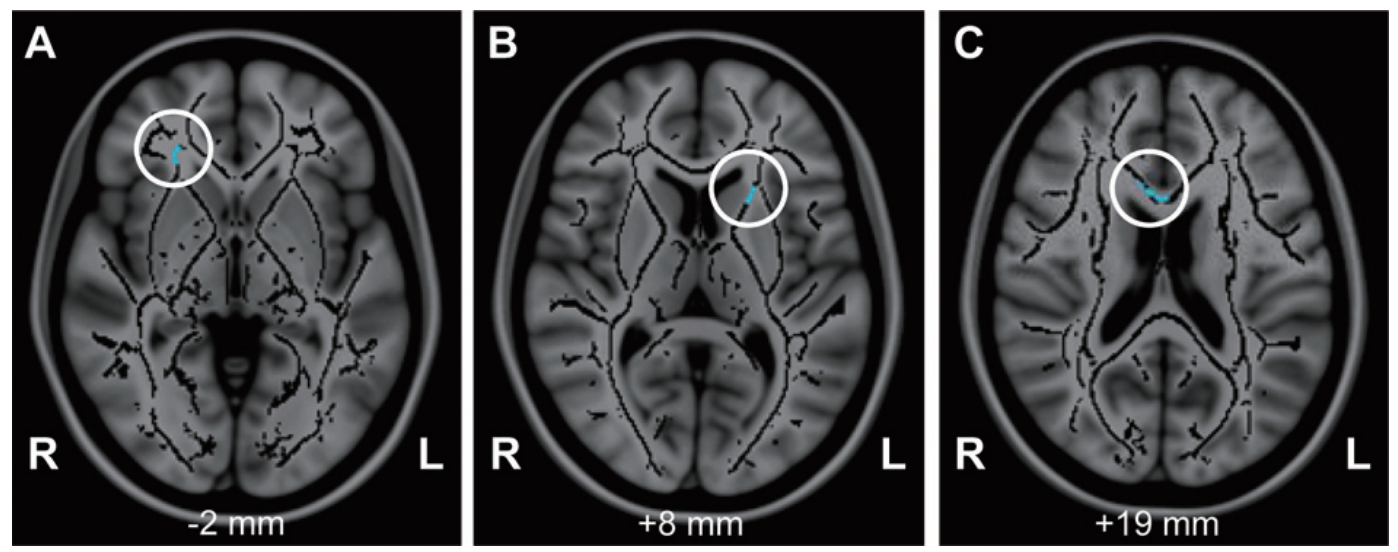

Fig. 4. Regions where FA was predictive of RBMT outcome from the I-MEMS memory therapy intervention one month post-therapy: (A) anterior region of corona radiata, (B) anterior limb of the internal capsule, and (C) rostral body of the corpus callosum. (Colours are visible in the online version of the article; http://dx.doi.org/10.3233/NRE-2012-0797)

ing that no individual data points had undue influence on the regression results. Finally, we re-ran the full models two additional times, first excluding individuals with mild injuries, and then (separately) excluding individuals with moderate injuries. Similar predictive regions were identified, and negative regression coefficients were obtained.

\section{Discussion}

We examined fractional anisotropy in participants with both chronic TBI and residual memory difficulties, along with a set of matched controls. We identified widespread reductions in FA associated with TBI, as has previously been found [7,48], as well as graded regional FA reductions associated with injury severity. We then identified distinct white matter regions that were predictive of outcome from a specific rehabilitation intervention, namely the I-MEMS intervention [41]. Regions with substantial predictive value were found both for an outcome closely related to the rehabilitation training (HVLT), as well as for a more functionally relevant outcome (RBMT). To our knowledge, this is the first report to use white matter integri- ty to predict outcomes from a particular rehabilitation intervention.

\subsection{FA changes with TBI}

The widespread reductions in FA associated with TBI (Fig. 2) - including the fornix, thalamus, and corpus callosum - are consistent with numerous previous results $[7,48,73,74,77]$ and with the notion that TBI is associated with widespread traumatic axonal injury and consequent neuronal degeneration. The substantial involvement of medial temporal lobe structures and connecting pathways is consistent with the memory difficulties observed in this group. The apparent left asymmetry of such FA reductions may be related to our group selection criteria. In particular, participants with TBI were included if they self-reported as having memory difficulties and such difficultly was also corroborated by a close friend or family member. The existence of memory difficulties was confirmed via neuropsychological testing. Neuropsychological testing as well as routine memory tasks are often based on verbal memory which is, in turn, strongly associated with left hemisphere function $[15,17,50]$. Hence, the leftward bias in FA reductions may reflect our inclusion 
criteria: individuals with residual memory impairment post-TBI may be more likely to have sustained white matter injury to the left hemisphere $[19,50]$.

\subsection{FA reductions as a function of injury severity}

We found that progressively more and larger regions of significantly reduced FA were identified at greater injury severities from mild to moderate to severe. Thus, as with more typical structural neuroimaging for clinical purposes, significant changes in WM are less apparent in mild and moderate TBI than in severe TBI.

Prior reports, mostly conducted in participants with severe TBI, have suggested that the TAI consequent to TBI results in uniformly decreased FA [24,29,38], similar to our findings. While it is possible that FA at the acute stage was also relatively uniformly decreased (we have no way of knowing in our group), FA for our chronic stage TBI participants revealed regional variation in the sign of the changes in FA consequent to injury. Binomial tests did not support the hypothesis of uniformly decreased FA, nor did it support the hypothesis of equal numbers of increases and decreases in FA. We consider this to indicate that the primary result of TAI is a reduction in FA, but that at least some ongoing neuroplastic changes occur that can regionally increase FA. In this way, we believe our findings provide limited support for the notion that structural remodeling, or neuroplasticity, may occur over the first several months or years post-injury [23,51]. Further research needs to be done to thoroughly test this hypothesis.

\subsection{Regional FA predicts rehabilitation outcome}

The primary focus of our study along with our novel prediction analysis procedure identified bilateral WM adjacent to the hippocampus as predictive of HVLT outcome at one month post-therapy, and three distinct prefrontal cortical regions as predictive of RBMT outcome at the same time point. Considering HVLT first, given the hippocampus' well known role in learning and memory $[11,56]$, it was expected that WM immediately adjacent to the hippocampus would exhibit a significant relationship with memory improvement following memory rehabilitation. Indeed, prior work has repeatedly demonstrated degeneration in the medial temporal lobe following TBI $[9,39]$, as well as an association between hippocampal damage and TBI outcome [66]. Our own work with fMRI identified the hippocampus (along with the DLPFC and the posterior parietal cortex) as important to performance of HVLT-like memo- ry tasks and which also exhibited decreased activity in individuals with TBI versus controls [58].

Concerning RBMT, we had previously found that functional activation in the ventrolateral prefrontal cortex provided predictive value for I-MEMS rehabilitation outcome [59]. Via structural neuroimaging, we additionally identified gray matter volumes in the cingulate gyrus, areas adjacent to the predicted VLPFC and PPC regions, and the thalamus as predictive of rehabilitation outcome [60]. Here, we identified: (i) FA in the rostral body of the corpus callosum, which has previously been implicated in TBI memory function [28], (ii) the anterior limb of the internal capsule which is connected with the thalamus and limbic system and is involved in attention to novel stimuli [40], as well as (iii) the anterior corona radiate, which broadly innervates the prefrontal cortex, but is specifically implicated in difficulties with executive control [78] and cognitive conflict such as remembering numerous different types of items [40]. Jointly these regions span a variety of executive control capabilities required for proper memory functioning in a more realistic and functional context - as the RBMT is intended to emulate. Thus, the regions identified in both the HVLT and RBMT prediction models are consistent with the cognitive demands generated by the respective tests.

Although the regional distribution of our findings were reasonably consonant with what is known about cerebral connectivity, the sign of the findings - namely that higher FA values predicted lower posttest memory scores in each region in Figs 3 and 4 - was unexpected. Negative coefficients were found for both HVLT as well as RBMT prediction. Importantly, the sign of the prediction held up not only in regions involving lower FA (parahippocampal WM) where FA is harder to estimate accurately, but also in major pathways with relatively high FA (the body of the corpus callosum). It also held up when excluding mild or moderate TBI participants from the analysis, and even when excluding other terms in the models.

Generally speaking, a simple Wallerian-like degeneration process is expected to decrease FA rather than increase FA. In a simplistic model, therefore, one might assume that TBI leads to TAI of WM tracts and a concomitant reduction of FA. Larger reductions in FA should then mark areas of greater damage, and areas with more damage are in turn presumed to more seriously affect learning, memory, executive, attentional or other behavioral performance. Given this logic, higher FA should be associated with higher post-test scores, the opposite of what we found. 
There is evidence, however, that this simple model may be incomplete. For example, Sidaros and colleagues [51] performed DTI in 30 individuals with severe TBI twice: at a mean of 8 -weeks and again at 12-months post-injury. They identified several regions where FA significantly increased over this interval. An explanation based on microstructural reorganization was provided, possibly including axonal regrowth in later stages after severe TBI. Regrowth could also help explain the considerable improvement in patient functioning over this same 12-month time period. A case study by Han and colleagues also demonstrated decreased FA at 12 weeks but increased FA at 24 months post-injury, with concomitant behavioral recovery [23]. Thus, rather than being static, it appears that FA can evolve over time following a TBI, including post-injury increases in FA. Our patients were all greater than one year post-injury (nearly 14 years postinjury, on average) and hence one would expect reorganization or repair processes were well underway or complete at the time of DTI scanning. The chronic TBI stage we investigated may therefore involve a mix of progressive or completed FA decreases in some WM pathways, alongside progressive or completed FA increases in other pathways.

The structural factors affecting FA include but are not limited to myelination, axon diameter, axon density, ultrastructure, and gliosis [5]. In addition, FA can be affected by fiber composition (e.g., crossing fibers) and partial volume effects where voxels containing fiber tracts along with gray matter, vessels, or other tissue of substantially different anisotropy [3]. While the TBSS registration process greatly reduces the likelihood of partial volume effects, other structural changes are indeed possible. Even in a seemingly simple context where one would expect faster response times to be associated with higher FA (i.e., higher neural conduction velocities), the opposite has been found [65]. The question remains as to how higher FA might be associated with poorer memory performance following the I-MEMS rehabilitation intervention.

We propose three potential hypotheses. One possibility is that the pre-injury white matter connectivity in the identified regions differed across participants, and this pre-injury FA is somehow related to HVLT and RBMT scores following the I-MEMS intervention. This could include various pre-injury factors including learning disability, prior brain insults, or normal variation that might correlate with overall learning and executive capacity necessary for successful response to cognitive rehabilitation. Since our healthy controls did not participate in the intervention or post-tests, we cannot rule out this possibility with the current study.

A second hypothesis is that injury initially occurred at these sites, but that some non-adaptive physiological process occurred that also increased FA. Possibilities here are limited, including chronic cytotoxic edema (not likely), gliotic changes that also increased FA (not likely), or perhaps increased water content in the myelin [34]. In this case, a poorer rehabilitation outcome with higher FA would simply reflect the extent of initial injury in these WM regions that appear key for HVLT or RBMT testing.

A third hypothesis is that injury initially occurred in the identified (predictive) regions, but that this was followed by a maladaptive physiological process such as inappropriate axonal regrowth. Such neuroplasticity would need to be associated with increased FA yet provide either ineffectual restoration, or inhibitory influence over the damaged pathways as in interhemispheric competition in recovery after stroke. Ineffectual restoration could be manifest as a region that contained damaged crossing fiber pathways being replaced by a single pathway. This might occur, for example, if those with chronic TBI used counterproductive strategies to compensate for memory deficits and partially regenerated ineffective or competing neural pathways. Interhemispheric competition has in fact been suggested by increased ipsilateral fMRI activity or EEG responses to stimulation $[20,33,37,69]$. Given that our identified regions have previously been suggested as important to the memory tasks used for outcome assessment, individuals with more maladaptation, and by this hypothesis higher FA, would thus suffer more interference and be less able to improve on the different types of tested memory functions using therapy-related strategies (internal rehearsal, elaboration and imagery). Differentiation of these hypotheses would require additional scanning time points, including comparable diffusion scans close to the time of injury.

\subsection{Clinical importance of neurorehabilitation outcome predictions}

Currently, it is difficult to custom-select rehabilitation interventions to treat the cognitive sequelae of TBI. Few studies provide guidance as to useful predictive factors, despite the cost and time required to implement and participate in such interventions. Identifying substantial and strong predictors or biomarkers for outcomes specific to particular domains of rehabilitation (i.e., memory vs. attention, physical vs. cognitive) 
could be quite beneficial. As with our previous neuroimaging findings, the prediction ranges afforded by FA rivaled those of pretest scores, even after accounting for variables such as pretest, age, and injury severity. This supports the notion that FA can indeed provide substantial added value for such predictions and hence may be clinically useful for predicting outcomes from specific rehabilitation interventions. It is presumed that the anatomical specificity provided by such measurements, and/or the sensitivity of such measurements to the underlying injury in TBI, as compared to radiological reports [8], provides key information about the disruptions to functional brain networks. Such information may be lost when simply considering, for example, overall injury severity based on behavioral markers, or total lesion load. To make maximal use of such a strategy will ultimately require similar neuroimaging information for multiple rehabilitation interventions, thereby enabling informed selection of, e.g., the optimal rehabilitation strategy based on a given patient's individual pattern of brain injury. If our hypotheses of ineffectual or maladaptive regrowth are supported by future studies, this would suggest the importance of providing interventions earlier - before such counterproductive processes begin to interfere with further rehabilitation potential.

\subsection{Study limitations}

We included individuals with a wide range of injury severities, and hence subgroups may have exhibited different responses from our "average" prediction coefficients. Similarly, our sample was variably chronic, so the time since injury and rehabilitation histories differed across participants. This variability almost certainly contributed to reduced (rather than inflated) prediction sensitivity. However, the variability does help support our study's generalizability: among individuals post-TBI who present with memory difficulties, the ability to predict memory rehabilitation outcome remained strong in spite of such heterogeneity, and hence the findings may apply to a relatively broad clinical population. It is possible that our approach is less sensitive if applied exclusively in mild TBI, where neurostructural changes may be less evident. It remains an open question whether our approach would be suitable for individuals who are less than one-year post-TBI.

While the (nearly) automated TBSS approach can greatly aid in alignment of FA maps across individuals, particularly in those with structural injury, the method in no way guarantees that the FA skeleton will map pre- cisely onto known tracts. Some immediately adjacent fiber bundles have highly disparate targets, yet their proximity results in a single "tract" in the FA skeleton. Localization of injury to a particular tract thus can be difficult without the use of tractography. Given the relatively thick slices and relatively few directions in our image acquisition and pilot analyses, we felt that fiber tracking would not be suitably reliable to pursue in this dataset. Overall, however, we believe that automated analysis support the potential clinical translation of the approach, by making analysis more feasible in larger groups and not requiring an expert neuroanatomist to read and highlight each scan.

\section{Conclusions}

We identified several specific regions in cerebral white matter that provided substantial predictive value for two different outcome measures following a memory rehabilitation intervention. While this represents only an early stage in predicting outcomes from specific cognitive interventions, and is specific to just one such intervention, the approach appears viable, and provides a benchmark with which to compare other neuroimaging (or neuropsychology or demographic) approaches to predicting rehabilitation outcomes. Since our study was conducted at the chronic stage, further work needs to be done to determine if more acute imaging provides better, worse or equivalent predictive value. However, if suitable outcome prediction can be achieved for this and other cognitive rehabilitation interventions, neuroimaging could eventually become a tool to help individuals with TBI and their caregivers more objectively select among candidate rehabilitation interventions based on post injury structural scans.

\section{Conflict of interest}

No commercial party having a direct financial interest in the results of the research supporting this article has or will confer a benefit upon the authors or upon any organization with which the authors are associated.

\section{Acknowledgements}

We would like to thank Dana Christina, Kalika Kelkar, the Statewide Head Injury Program, and Community Rehabilitation Care for their help and support. 
The contents of this publication were developed under a grant from the National Institute on Disability and Rehabilitation Research, Department of Education (H133A020513). However, those contents do not necessarily represent the policy of the Department of Education, and you should not assume endorsement by the Federal Government. Support for this project also came from the NIH (NINDS: K25-NS046554).

\section{References}

[1] Arfanakis K, Haughton VM, Carew JD, Rogers BP, Dempsey RJ, Meyerand ME (2002) Diffusion tensor MR imaging in diffuse axonal injury. AJNR Am J Neuroradiol 23:794-802.

[2] Ariza M, Serra-Grabulosa JM, Junque C, Ramirez B, Mataro M, Poca A, Bargallo N, Sahuquillo J (2006) Hippocampal head atrophy after traumatic brain injury. Neuropsychologia 44:1956-1961.

[3] Assaf Y, Pasternak O (2008) Diffusion tensor imaging (DTI)based white matter mapping in brain research: A review. J Mol Neurosci 34:51-61.

[4] Azouvi P (2000) Neuroimaging correlates of cognitive and functional outcome after traumatic brain injury. Curr Opin Neurol 13:665-669.

[5] Beaulieu C (2002) The basis of anisotropic water diffusion in the nervous system - a technical review. NMR Biomed 15:435-455.

[6] Bendlin BB, Ries ML, Lazar M, Alexander AL, Dempsey RJ, Rowley HA, Sherman JE, Johnson SC (2008) Longitudinal changes in patients with traumatic brain injury assessed with diffusion-tensor and volumetric imaging. Neuroimage 42:503514.

[7] Benson RR, Meda SA, Vasudevan S, Kou Z, Govindarajan KA, Hanks RA, Millis SR, Makki M, Latif Z, Coplin W, Meythaler J, Haacke EM (2007) Global white matter analysis of diffusion tensor images is predictive of injury severity in traumatic brain injury. J Neurotrauma 24:446-459.

[8] Bigler ED (2001) Quantitative magnetic resonance imaging in traumatic brain injury. J Head Trauma Rehabil 16:117-134.

[9] Bigler ED, Maxwell WL (2011) Neuroimaging and neuropathology of TBI. NeuroRehabilitation 28:63-74.

[10] Brandt J, Benedict RHB (2001) Hopkins Verbal Learning TestRevised: Psychological Assessment Resources.

[11] Burgess N, Maguire EA, O'Keefe J (2002) The human hippocampus and spatial and episodic memory. Neuron 35:625641.

[12] Cicerone K, Levin H, Malec J, Stuss D, Whyte J (2006) Cognitive rehabilitation interventions for executive function: moving from bench to bedside in patients with traumatic brain injury. J Cogn Neurosci 18:1212-1222.

[13] Cicerone KD, Dahlberg C, Kalmar K, Langenbahn DM, Malec JF, Bergquist TF, Felicetti T, Giacino JT, Harley JP, Harrington DE, Herzog J, Kneipp S, Laatsch L, Morse PA (2000) Evidence-based cognitive rehabilitation: recommendations for clinical practice. Arch Phys Med Rehabil 81:1596-1615.

[14] Cicerone KD, Mott T, Azulay J, Friel JC (2004) Community integration and satisfaction with functioning after intensive cognitive rehabilitation for traumatic brain injury. Arch Phys Med Rehabil 85:943-950.
[15] Coughlan AK (1979) Effects of localised cerebral lesions and dysphasia on verbal memory. J Neurol Neurosurg Psychiatry 42:914-923.

[16] Dikmen SS, Machamer JE, Powell JM, Temkin NR (2003) Outcome 3 to 5 years after moderate to severe traumatic brain injury. Arch Phys Med Rehabil 84:1449-1457.

[17] Faust M, Ben-Artzi E, Harel I (2008) Hemispheric asymmetries in semantic processing: evidence from false memories for ambiguous words. Brain Lang 105:220-228.

[18] Filley CM (2005a) Neurobehavioral aspects of cerebral white matter disorders. Psychiatr Clin North Am 28:685-700, 697688.

[19] Filley CM (2005b) White matter and behavioral neurology. Ann N Y Acad Sci 1064:162-183.

[20] Gerloff C, Bushara K, Sailer A, Wassermann EM, Chen R, Matsuoka T, Waldvogel D, Wittenberg GF, Ishii K, Cohen LG, Hallett M (2006) Multimodal imaging of brain reorganization in motor areas of the contralesional hemisphere of well recovered patients after capsular stroke. Brain 129:791-808.

[21] Giles GM (2001) The effectiveness of neurorehabilitation. In: Neurobehavioral disability and social handicap following traumatic brain injury(Wood, R. L. and McMillan, T. M., eds), pp 231-255 Hove, England: Psychology Press.

[22] Greenberg G, Mikulis DJ, Ng K, DeSouza D, Green RE (2008) Use of diffusion tensor imaging to examine subacute white matter injury progression in moderate to severe traumatic brain injury. Arch Phys Med Rehabil 89:S45-50.

[23] Han BS, Kim SH, Kim OL, Cho SH, Kim YH, Jang SH (2007) Recovery of corticospinal tract with diffuse axonal injury: a diffusion tensor image study. NeuroRehabilitation 22:151155.

[24] Inglese M, Makani S, Johnson G, et al. Diffuse axonal injury in mild traumatic brain injury: A diffusion tensor imaging study. J Neurosurg 2005;103:298-303.

[25] Jorge RE, Acion L, Starkstein SE, Magnotta V (2007) Hippocampal volume and mood disorders after traumatic brain injury. Biol Psychiatry 62:332-338.

[26] Katz DI, Alexander MP (1994) Traumatic brain injury. Predicting course of recovery and outcome for patients admitted to rehabilitation. Arch Neurol 51:661-670.

[27] Kim YH, Yoo WK, Ko MH, Park CH, Kim ST, Na DL (2009) Plasticity of the attentional network after brain injury and cognitive rehabilitation. Neurorehabil Neural Repair 23:468477.

[28] Kraus MF, Susmaras T, Caughlin BP, Walker CJ, Sweeney JA, Little DM (2007) White matter integrity and cognition in chronic traumatic brain injury: a diffusion tensor imaging study. Brain 130:2508-2519.

[29] Kumar R, Husain M, Gupta RK, et al. Serial changes in the white matter diffusion tensor imaging metrics in moderate traumatic brain injury and correlation with neuro-cognitive function. J Neurotrauma 2009;26:481-495.

[30] Levin HS (2003) Neuroplasticity following non-penetrating traumatic brain injury. Brain Inj 17:665-674.

[31] Levin HS, Wilde EA, Chu Z, Yallampalli R, Hanten GR, Li X, Chia J, Vasquez AC, Hunter JV (2008) Diffusion tensor imaging in relation to cognitive and functional outcome of traumatic brain injury in children. J Head Trauma Rehabil 23:197-208.

[32] Ljungqvist J, Nilsson D, Ljungberg M, Sorbo A, Esbjornsson E, Eriksson-Ritzen C, Skoglund T (2011) Longitudinal study of the diffusion tensor imaging properties of the corpus callosum in acute and chronic diffuse axonal injury. Brain Inj 25:370-378. 
[33] Lotze M, Markert J, Sauseng P, Hoppe J, Plewnia C, Gerloff C (2006) The role of multiple contralesional motor areas for complex hand movements after internal capsular lesion. J Neurosci 26:6096-6102.

[34] Mayer AR, Ling J, Mannell MV, Gasparovic C, Phillips JP, Doezema D, Reichard R, Yeo RA (2010) A prospective diffusion tensor imaging study in mild traumatic brain injury. Neurology 74:643-650.

[35] McAllister TW, Flashman LA, McDonald BC, Saykin AJ (2006) Mechanisms of working memory dysfunction after mild and moderate TBI: evidence from functional MRI and neurogenetics. J Neurotrauma 23:1450-1467.

[36] Merkley TL, Bigler ED, Wilde EA, McCauley SR, Hunter JV, Levin HS (2008) Diffuse changes in cortical thickness in pediatric moderate-to-severe traumatic brain injury. J Neurotrauma 25:1343-1345.

[37] Nair DG, Hutchinson S, Fregni F, Alexander M, PascualLeone A, Schlaug G (2007) Imaging correlates of motor recovery from cerebral infarction and their physiological significance in well-recovered patients. Neuroimage 34:253-263.

[38] Newcombe V, Chatfield D, Outtrim J, Vowler S, Manktelow A, Cross J, Scoffings D, Coleman M, Hutchinson P, Coles J, Carpenter TA, Pickard J, Williams G, Menon DK (2010) Mapping Traumatic Axonal Injury Using Diffusion Tensor Imaging: Correlations with Functional Outcome. PLoS ONE 6:e19214.

[39] Ng K, Mikulis DJ, Glazer J, Kabani N, Till C, Greenberg G, Thompson A, Lazinski D, Agid R, Colella B, Green RE (2008) Magnetic resonance imaging evidence of progression of subacute brain atrophy in moderate to severe traumatic brain injury. Arch Phys Med Rehabil 89:S35-44.

[40] Niogi S, Mukherjee P, Ghajar J, McCandliss BD (2010) Individual Differences in Distinct Components of Attention are Linked to Anatomical Variations in Distinct White Matter Tracts. Front Neuroanat 4:2.

[41] O’Neil-Pirozzi TM, Strangman GE, Goldstein R, Katz DI, Savage CR, Kelkar K, Supelana C, Burke D, Rauch SL, Glenn MB (2010) A controlled treatment study of internal memory strategies (I-MEMS) following traumatic brain injury. J Head Trauma Rehabil 25:43-51.

[42] Oldfield R (1971) The assessment and analysis of handedness. The Edinburgh inventory. Neuropsychologia 9:97-113.

[43] Palacios EM, Fernandez-Espejo D, Junque C, SanchezCarrion R, Roig T, Tormos JM, Bargallo N, Vendrell P (2011) Diffusion tensor imaging differences relate to memory deficits in diffuse traumatic brain injury. BMC Neurol 11:24.

[44] Perlbarg V, Puybasset L, Tollard E, Lehericy S, Benali H, Galanaud D (2009) Relation between brain lesion location and clinical outcome in patients with severe traumatic brain injury: a diffusion tensor imaging study using voxel-based approaches. Hum Brain Mapp 30:3924-3933.

[45] Povlishock JT, Katz DI (2005) Update of neuropathology and neurological recovery after traumatic brain injury. J Head Trauma Rehabil 20:76-94.

[46] Rueckert D, Sonoda LI, Hayes C, Hill DL, Leach MO, Hawkes DJ (1999) Nonrigid registration using free-form deformations: application to breast MR images. IEEE Trans Med Imaging 18:712-721.

[47] Ruff RM, Marshall LF, Crouch J, Klauber MR, Levin HS, Barth J, Kreutzer J, Blunt BA, Foulkes MA, Eisenberg HM, et al. (1993) Predictors of outcome following severe head trauma: follow-up data from the Traumatic Coma Data Bank. Brain Inj 7:101-111.
[48] Salmond CH, Chatfield DA, Menon DK, Pickard JD, Sahakian BJ (2005) Cognitive sequelae of head injury: involvement of basal forebrain and associated structures. Brain 128:189-200.

[49] Salmond CH, Menon DK, Chatfield DA, Williams GB, Pena A, Sahakian BJ, Pickard JD (2006) Diffusion tensor imaging in chronic head injury survivors: correlations with learning and memory indices. Neuroimage 29:117-124.

[50] Sepulcre J, Masdeu JC, Sastre-Garriga J, Goni J, Velez-deMendizabal N, Duque B, Pastor MA, Bejarano B, Villoslada $P$ (2008) Mapping the brain pathways of declarative verbal memory: Evidence from white matter lesions in the living human brain. Neuroimage 42:1237-1243.

[51] Sidaros A, Engberg AW, Sidaros K, Liptrot MG, Herning M, Petersen P, Paulson OB, Jernigan TL, Rostrup E (2008) Diffusion tensor imaging during recovery from severe traumatic brain injury and relation to clinical outcome: A longitudinal study. Brain 131:559-572.

[52] Smith SM (2002) Fast robust automated brain extraction. Hum Brain Mapp 17:143-155.

[53] Smith SM, Jenkinson M, Johansen-Berg H, Rueckert D, Nichols TE, Mackay CE, Watkins KE, Ciccarelli O, Cader MZ, Matthews PM, Behrens TE (2006) Tract-based spatial statistics: voxelwise analysis of multi-subject diffusion data. Neuroimage 31:1487-1505.

[54] Smith SM, Jenkinson M, Woolrich MW, Beckmann CF, Behrens TE, Johansen-Berg H, Bannister PR, De Luca M, Drobnjak I, Flitney DE, Niazy RK, Saunders J, Vickers J, Zhang Y, De Stefano N, Brady JM, Matthews PM (2004) Advances in functional and structural MR image analysis and implementation as FSL. Neuroimage 23 Suppl 1:S208-219.

[55] Smith SM, Nichols TE (2009) Threshold-free cluster enhancement: addressing problems of smoothing, threshold dependence and localisation in cluster inference. Neuroimage 44:8398.

[56] Squire LR (1992) Memory and the hippocampus: A synthesis from findings with rats, monkeys and humans. Psychological Review 99:195-231.

[57] Strangman G, O’Neil-Pirozzi TM, Burke D, Cristina D, Goldstein R, Rauch SL, Savage CR, Glenn MB (2005) Functional neuroimaging and cognitive rehabilitation for people with traumatic brain injury. Am J Phys Med Rehabil 84:62-75.

[58] Strangman GE, Goldstein R, O'Neil-Pirozzi TM, Kelkar K, Supelana C, Burke D, Katz DI, Rauch SL, Savage CR, Glenn MB (2009a) Neurophysiological alterations during strategybased verbal learning in traumatic brain injury. Neurorehabil Neural Repair 23:226-236.

[59] Strangman GE, O’Neil-Pirozzi TM, Goldstein R, Kelkar K, Katz DI, Burke D, Rauch SL, Savage CR, Glenn MB (2008) Prediction of memory rehabilitation outcomes in traumatic brain injury by using functional magnetic resonance imaging. Arch Phys Med Rehabil 89:974-981.

[60] Strangman GE, O’Neil-Pirozzi TM, Supelana C, Goldstein R, Katz DI, Glenn MB (2010) Regional brain morphometry predicts memory rehabilitation outcome after traumatic brain injury. Front Hum Neurosci 4:182.

[61] Strangman GE, Zhang Q, Zeffiro T (2009b) Near-Infrared Neuroimaging with NinPy. Front Neuroinformatics 3:12.

[62] Sugiyama K, Kondo T, Oouchida Y, Suzukamo Y, Higano S, Endo M, Watanabe H, Shindo K, Izumi S (2009) Clinical utility of diffusion tensor imaging for evaluating patients with diffuse axonal injury and cognitive disorders in the chronic stage. J Neurotrauma 26:1879-1890.

[63] Temkin NR, Holubkov R, Machamer JE, Winn HR, Dikmen SS (1995) Classification and regression trees (CART) for pre- 
diction of function at 1 year following head trauma. J Neurosurg 82:764-771.

[64] Tollard E, Galanaud D, Perlbarg V, Sanchez-Pena P, Le Fur Y, Abdennour L, Cozzone P, Lehericy S, Chiras J, Puybasset L (2009) Experience of diffusion tensor imaging and $1 \mathrm{H}$ spectroscopy for outcome prediction in severe traumatic brain injury: Preliminary results. Crit Care Med 37:1448-1455.

[65] Tuch DS, Salat DH, Wisco JJ, Zaleta AK, Hevelone ND, Rosas HD (2005) Choice reaction time performance correlates with diffusion anisotropy in white matter pathways supporting visuospatial attention. Proc Natl Acad Sci U S A 102:1221212217.

[66] Umile EM, Sandel ME, Alavi A, Terry CM, Plotkin RC (2002) Dynamic imaging in mild traumatic brain injury: support for the theory of medial temporal vulnerability. Arch Phys Med Rehabil 83:1506-1513.

[67] Wang JY, Abdi H, Bakhadirov K, Diaz-Arrastia R, Devous MD, Sr. (2011) A comprehensive reliability assessment of quantitative diffusion tensor tractography. Neuroimage.

[68] Wang JY, Bakhadirov K, Devous MD, Sr., Abdi H, McColl R, Moore C, Marquez de la Plata CD, Ding K, Whittemore A, Babcock E, Rickbeil T, Dobervich J, Kroll D, Dao B, Mohindra N, Madden CJ, Diaz-Arrastia R (2008) Diffusion tensor tractography of traumatic diffuse axonal injury. Arch Neurol 65:619-626.

[69] Ward NS, Brown MM, Thompson AJ, Frackowiak RS (2003) Neural correlates of outcome after stroke: a cross-sectional fMRI study. Brain 126:1430-1448.

[70] Wei CW, Tharmakulasingam J, Crawley A, Kideckel DM, Mikulis DJ, Bradbury CL, Green RE (2008) Use of diffusiontensor imaging in traumatic spinal cord injury to identify concomitant traumatic brain injury. Arch Phys Med Rehabil 89:S85-91

[71] Wilde EA, Chu Z, Bigler ED, Hunter JV, Fearing MA, Hanten G, Newsome MR, Scheibel RS, Li X, Levin HS (2006) Di- ffusion tensor imaging in the corpus callosum in children after moderate to severe traumatic brain injury. J Neurotrauma 23:1412-1426.

[72] Wilde EA, Hunter JV, Newsome MR, Scheibel RS, Bigler ED, Johnson JL, Fearing MA, Cleavinger HB, Li X, Swank PR, Pedroza C, Roberson GS, Bachevalier J, Levin HS (2005) Frontal and temporal morphometric findings on MRI in children after moderate to severe traumatic brain injury. J Neurotrauma 22:333-344.

[73] Wilde EA, McCauley SR, Hunter JV, Bigler ED, Chu Z, Wang ZJ, Hanten GR, Troyanskaya M, Yallampalli R, Li X, Chia J, Levin HS (2008) Diffusion tensor imaging of acute mild traumatic brain injury in adolescents. Neurology 70:948-955.

[74] Wilde EA, Newsome MR, Bigler ED, Pertab J, Merkley TL, Hanten G, Scheibel RS, Li X, Chu Z, Yallampalli R, Hunter JV, Levin HS (2011) Brain imaging correlates of verbal working memory in children following traumatic brain injury. Int $\mathrm{J}$ Psychophysiol 82:86-96.

[75] Wilde EA, Ramos MA, Yallampalli R, Bigler ED, McCauley SR, Chu Z, Wu TC, Hanten G, Scheibel RS, Li X, Vasquez AC, Hunter JV, Levin HS (2010) Diffusion tensor imaging of the cingulum bundle in children after traumatic brain injury. Dev Neuropsychol 35:333-351.

[76] Wilson B, Cockburn J, Baddeley A, Hiorns R (2003) The Rivermead Behavioural Memory Test-II Supplement Two. Bury St. Edmunds: Thames Valley Test Company.

[77] Xu J, Rasmussen IA, Lagopoulos J, Haberg A (2007) Diffuse axonal injury in severe traumatic brain injury visualized using high-resolution diffusion tensor imaging. J Neurotrauma 24:753-765.

[78] Yin X, Han Y, Ge H, Xu W, Huang R, Zhang D, Xu J, Fan L, Pang Z, Liu S (2011) Inferior frontal white matter asymmetry correlates with executive control of attention. Hum Brain Mapp. 